\title{
Coherent Light Emission from Planar Plasmonic Metamaterials
}

\author{
G. Adamo ${ }^{1 *}$, J. Y. Ou ${ }^{1}$, J. K. So ${ }^{1}$, M. Ren ${ }^{1,2}$, E. Plum ${ }^{1}$, E. T. F. Rogers ${ }^{1}$, K. F. MacDonald ${ }^{1}$, J. Xu ${ }^{2}$, \\ and N. I. Zheludev 1 \\ 1. Optoelectronics Research Centre \& Centre for Photonics Metamaterials, University of Southampton, SO17 1BJ, United Kingdom \\ 2. Key Laboratory of Weak Light Nonlinear Photonics, Nankai University, Tianjin 300457, P.R. China \\ *gia@orc.soton.ac.uk
}

\begin{abstract}
We show experimentally that highly localized excitations in planar plasmonic metamaterials drive spatially-coherent, directional, threshold-free light emission, providing a platform for the development of a new generation of nanoscale light sources.

OCIS codes: (160.3918) Metamaterials; (240.6680) Surface plasmons; (030.1670) Coherent optical effects
\end{abstract}

We report on the experimental demonstration of a fundamentally new nanostructural radiation phenomenon: In 'coherent' metamaterials, where interactions among meta-molecules are strong, the localized excitation of a single meta-molecule can generate a synchronized, in-phase response across a large number of meta-molecules, leading to threshold-free emission of spatially coherent light (Fig. 1a). Free-electron and tightly-focused optical excitations are found to drive resonant emission from planar plasmonic metamaterials over meta-molecule ensembles many times larger than the energy injection spot. The emission is linked to the collective mode underpinning the metamaterial absorption resonance and displays a collapse in linewidth as the number of emitting meta-molecules increases.
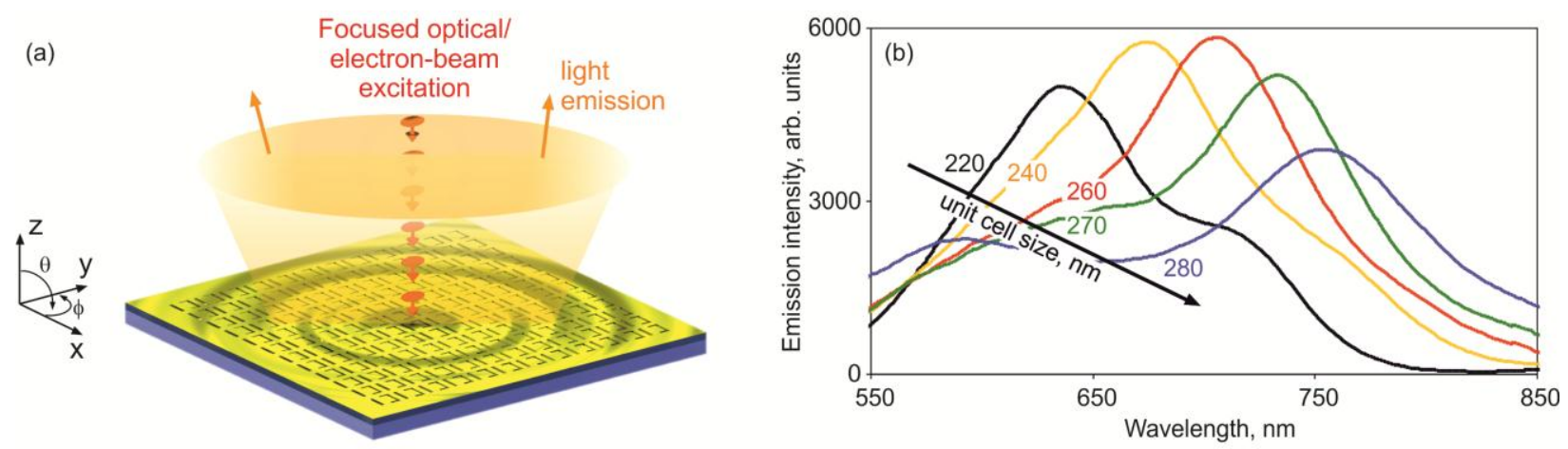

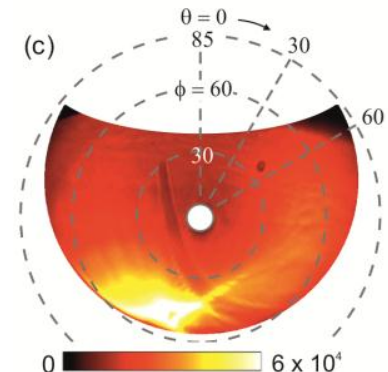

Total emission

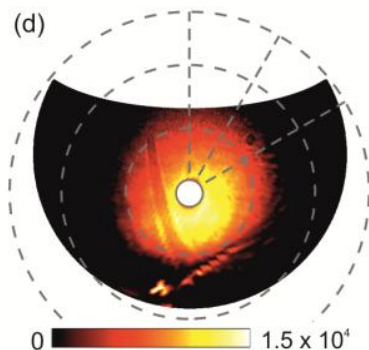

MM total emission

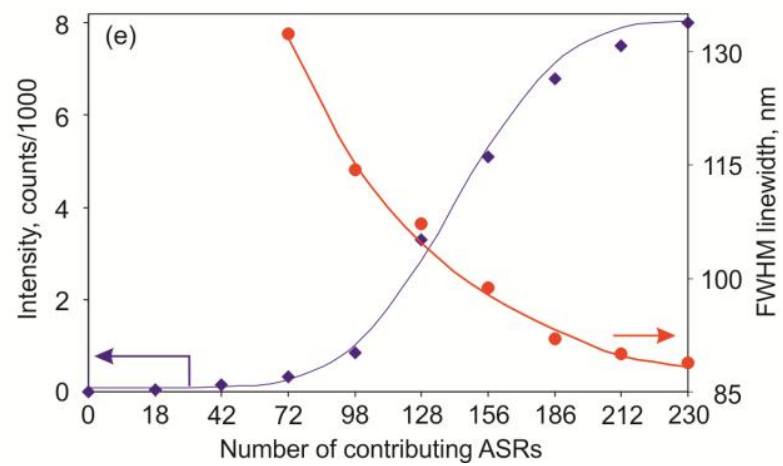

Fig. 1: (a) Localized electron-beam or optical excitation of a metamaterial array leads to the coherent oscillation of many cells and emission of light in the surface-normal direction; (b) Electron-beam-induced metamaterial light emission spectra for arrays with different meta-molecule cell sizes (as labeled); (c, d) Angular intensity distributions of total electron-induced light emission from a metamaterial array with a $220 \mathrm{~nm}$ unit cell integrated over all wavelengths from 400 to $1100 \mathrm{~nm}$ and the metamaterial-specific component of this spectrally integrated emission (background contribution from unstructured gold removed). (e) Intensity and half-maximum width of the $640 \mathrm{~nm}$ metamaterial emission line as a function of the number of contributing meta-molecules. 
In a laser, coherence is derived from the bosonic statistics of photons, the resonant properties of the laser cavity and the collective nature of stimulated emission in the gain medium, and a threshold level of input energy is required to maintain the gain and overcome losses. However, in an optical source constructed on the basis of a collectively oscillating optical nano-antenna (metamaterial) array it is provided not by the feed (as in conventional microwave antenna arrays) but via the strong mutual interactions among the nano-antennas.

Our experiments employ coherent metamaterial arrays of asymmetrically spit rings (ASRs) with Fano-like collective plasmonic modes manufactured by focused ion beam milling in $50 \mathrm{~nm}$ thick gold films. Electron-induced light emission spectra were recorded in a scanning electron microscope equipped with achromatic reflective lightcollection optics and a spectrometer with a liquid nitrogen-cooled CCD array detector. These reveal a correlation between the spectral positions of electron-induced light emission peaks and meta-molecule cell size (Fig. 1b), directly linking metamaterial emission to the collective mode underpinning the metamaterials' plasmonic absorption resonance.

Emission directionality was studied by projecting the light radiated by samples onto the CCD. These measurements clearly demonstrate that in contrast to the total broadband emission of unstructured gold, metamaterial emission at the plasmon resonance wavelength is highly directional (Figs. 1c, d). The low divergence of the resonant metamaterial emission results from its spatially coherent nature across a large number of metamolecules and is strongly indicative of the collective nature of the underlying excitation mode.

It is known that the width of the absorption peak in a finite ASR metamaterial array collapses with increasing array size (a behavior reflective of the collective, coherent nature of the plasmonic mode behind the resonant absorption) and experiments reveal a corresponding collapse in the emission linewidth as the number of contributing meta-molecules increases (Fig. 1e).

A complementary study of optically-pumped (photo-luminescent) emission from the same type of metamaterial further demonstrates that a highly localized input (400 nm laser light focused to a spot with a $\mathrm{e}^{-1}$ diameter of 350 $\mathrm{nm}, \sim 1.5$ unit cell widths) can generate a collective oscillation among many meta-molecules and drive resonant photo-luminescent emission over a much larger area than the excitation spot. Indeed, at the plasmonic resonance an emission spot diameter as big as $\sim 1100 \mathrm{~nm}$ was observed (Fig. 2).

(a)

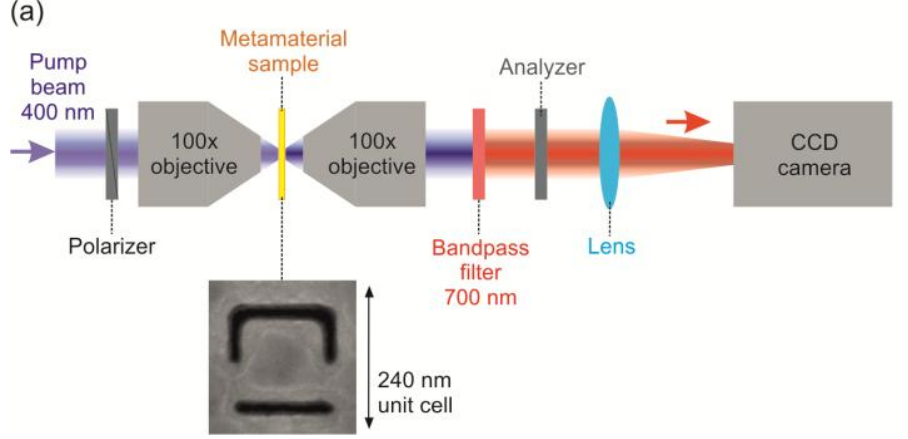

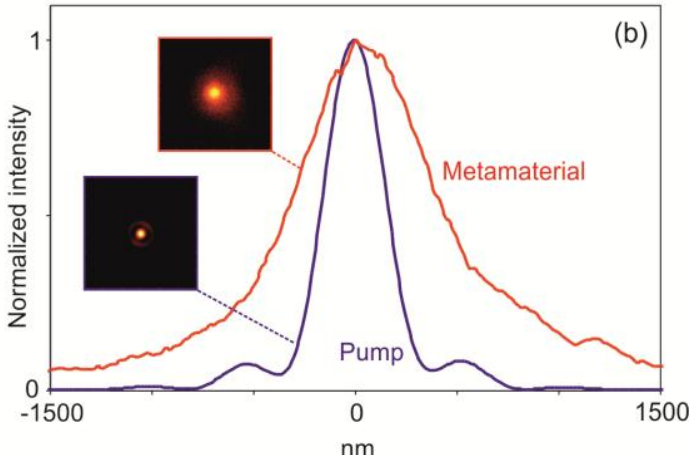

Fig. 2: (a) Experimental configuration for analysis of metamaterial photoluminescence. Pump light is focused onto the sample by a microscope objective. A second objective collects luminescent emission at a wavelength of $700 \mathrm{~nm}$ in the forward direction. The inset shows an SEM image a metamaterial unit cell; (b) Normalized intensity profiles and CCD images of the input pump beam [350 $\mathrm{nm}$ diameter] and output photo-luminescence spot [1100 nm].

Collective oscillations in planar plasmonic metamaterials may be driven by optical, electronic, plasmonic or electron-beam inputs and as such offer a generic platform for the development of new kinds of scalable, thresholdfree, spatially coherent light sources. 